\title{
Cocaine-evoked negative symptoms require AMPA receptor trafficking in the lateral habenula
}

\begin{abstract}
Frank J Meye $e^{1-3,8}$, Kristina Valentinova ${ }^{1-3,8}$, Salvatore Lecca ${ }^{1-3,8}$, Lucile Marion-Poll1-3, Matthieu J Maroteaux ${ }^{1-3}$, Stefano Musardo ${ }^{4}$, Imane Moutkine ${ }^{1-3}$, Fabrizio Gardoni ${ }^{4}$, Richard L Huganir ${ }^{5}$, François Georges ${ }^{6,7}$ \& Manuel Mameli ${ }^{1-3}$

Addictive substances mediate positive and negative states promoting persistent drug use. However, substrates for aversive effects of drugs remain elusive. We found that, in mouse lateral habenula (LHb) neurons targeting the rostromedial tegmental nucleus, cocaine enhanced glutamatergic transmission, reduced $\mathrm{K}^{+}$currents and increased excitability. GluA1 trafficking in LHb was instrumental for these cocaine-evoked modifications and drug-driven aversive behaviors. Altogether, our results suggest that long-lasting adaptations in LHb shape negative symptoms after drug taking.
\end{abstract}

Withdrawal from addictive substances, including cocaine, produces negative symptoms such as a depressive-like phenotype that contributes to compulsive drug abuse ${ }^{1,2}$. The lateral habenula (LHb) inhibits monoaminergic systems via the GABAergic rostromedial tegmental nucleus (RMTg), encoding aversion-related stimuli ${ }^{3,4}$. Notably, functional modifications of the LHb are associated with neuropsychiatric disorders, including addiction and depression ${ }^{5-7}$. However, whether a drug-mediated depressive-like phenotype is a consequence of drug-evoked adaptations in the habenulo-mesencephalic pathway remains unknown.

We examined the effects of cocaine on glutamatergic synaptic transmission onto mouse retrogradely labeled RMTg-projecting LHb neurons (LHb $\rightarrow$ RMTg; Fig. 1a and Supplementary Fig. 1a,b). Miniature excitatory postsynaptic current (mEPSC) amplitudes, but not frequencies, were larger in $\mathrm{LHb} \rightarrow \mathrm{RMTg}$ neurons $24 \mathrm{~h}$ after two consecutive cocaine injections $(20 \mathrm{mg}$ per $\mathrm{kg}$ of body weight, intraperitoneal; Fig. $\mathbf{1 b}, \mathbf{c})^{6}$. This cocaine-induced synaptic potentiation persisted 1 week after cocaine exposure, as well as $14 \mathrm{~d}$ after a chronic cocaine regimen (Supplementary Fig. 1c,d). This time course parallels the emergence of strong negative symptoms during drug-free periods ${ }^{1}$. Drug-evoked plasticity was detected only in LHb $\rightarrow$ RMTg, but not LHb neurons sending axons to the ventral tegmental area ( $\mathrm{LHb}^{\mathrm{V}} \rightarrow \mathrm{VTA}$; Supplementary Fig. 1e). This suggests that cocaine promotes a projection-specific and enduring postsynaptic strengthening of excitatory transmission onto $\mathrm{LHb}^{\mathrm{RMMTg}}$ neurons. However, whether cocaine alters LHb subpopulations other than RMTg/VTA-projecting neurons remains to be tested.
LHb synapses largely express GluA1-containing rectifying AMPARs ${ }^{8}$. Based on this subunit composition, we tested the necessity of GluA1 trafficking for cocaine-induced synaptic potentiation. We designed a viral vector encoding a dominant-negative form of the GluA1 C terminus (GluAlct) to temporally prevent activity-dependent synaptic delivery of GluA1 (ref. 9) via doxycycline-driven recombination ${ }^{10}$. Doxycycline administration led to GluAlct expression solely in the LHb (Fig. 1d and Supplementary Fig. If,g). We performed consecutive recordings from neighboring LHb $\rightarrow$ RMTg neurons infected or uninfected with GluA1ct (Fig. 1d and Online Methods). GluA1ct overexpression left basal transmission unaltered (Fig. 1e,f) ${ }^{9}$. After cocaine administration, mEPSC amplitude was increased in GluAlct-uninfected LHb $\rightarrow$ RMTg neurons. Notably, in neighboring neurons expressing GluAlct, the cocaineevoked potentiation was prevented (Fig. 1f). Accordingly, cocaine increased evoked AMPAR-EPSCs $(-70 \mathrm{mV})$ and AMPAR-NMDAR ratios (+50 mV; Fig. 1g,h and Supplementary Fig. $1 \mathbf{h}, \mathbf{i})$, which was precluded in $\mathrm{LHb} \rightarrow \mathrm{RMTg}$ neurons expressing GluAlct. We detected no substantial change in EPSC rectification index, suggesting that, despite the insertion of new AMPARs at the synapse, the overall AMPAR subunit composition remained similar after cocaine treatment (Supplementary Fig. 1j).

The phosphorylation of serine 845 (S845) and serine 831 (S831) on the GluA1 C terminus crucially controls AMPAR membrane delivery in various structures ${ }^{11}$. Immunoblots from microdissected LHbs revealed that phosphorylation of S845 was markedly increased following cocaine treatment. We observed no modifications of phosphoS831 or total GluA1 (Supplementary Fig. 2a,b). Accordingly, S845 phospho-mutant mice (S845A) showed no cocaine-induced mESPC amplitude enhancement, whereas this plasticity in S831 phosphomutant mice (S831A) was left intact (Supplementary Fig. 2c-f). Thus, cocaine drives $\mathrm{S} 845$ phosphorylation-dependent GluA1 delivery onto LHb $\rightarrow$ RMTg neurons.

We assessed the importance of cocaine exposure on the in vivo firing rate of LHb $\rightarrow$ RMTg neurons in anesthetized mice (Fig. 2a and Supplementary Fig. 2g). LHb $\rightarrow$ RMTg neuronal firing rate was higher in cocaine- than saline-treated mice (Figs. 1c and $\mathbf{2 b}, \mathbf{c}$ ). Given that single-cell GluA1ct expression is difficult to assess in vivo, we evaluated the necessity of GluA1 trafficking for $\mathrm{LHb} \rightarrow \mathrm{RMTg}$ neuronal hyperexcitability in acute brain slices. Cocaine increased the excitability of LHb $\rightarrow$ RMTg neurons not expressing GluA1ct. However, excitability in GluA1ct-infected LHb $\rightarrow$ RMTg neurons was comparable to that of saline controls (Figs. If and 2d,e). Similar to the synaptic adaptations, hyperexcitability was still present $14 \mathrm{~d}$ after chronic cocaine exposure (Fig. 2g,h and Supplementary Fig. 2h). Notably, blockade of AMPARs by NBQX in slices did not affect cocaine-evoked hyperexcitability, suggesting a causal, but indirect, effect of GluA1

\footnotetext{
${ }^{1}$ Institut du Fer à Moulin, Paris, France. ${ }^{2}$ Inserm, UMR-S 839, Paris, France. ${ }^{3}$ Université Pierre et Marie Curie, Paris, France. ${ }^{4}$ DiSFeB, Dipartimento di Scienze Farmacologiche e Biomolecolari, Università degli Studi di Milano, Milano, Italy. ${ }^{5}$ Solomon H. Snyder Department of Neuroscience, The Johns Hopkins University School of Medicine, Baltimore, Maryland, USA. ${ }^{6}$ Centre National de la Recherche Scientifique, Interdisciplinary Institute for Neuroscience, UMR 5297, Bordeaux, France. ${ }^{7}$ Université de Bordeaux, Bordeaux, France. ${ }^{8}$ These authors contributed equally to this work. Correspondence should be addressed to M.M. (manuel.mameli@inserm.fr). 
Figure 1 Cocaine-evoked synaptic potentiation onto $\mathrm{LHb} \rightarrow \mathrm{RMTg}$ neurons requires GluA1 delivery. (a) Schematic and images indicating the retrograde labeling of $\mathrm{LHb} \rightarrow \mathrm{RMTg}$ neurons. (b) Sample mEPSCs from LHb $\rightarrow$ RMTg neurons. (c) Scatter plot and grouped data for mEPSC frequency and amplitude ( $n=10-12$ cells, 4 mice of 4-5 weeks; frequency, saline versus cocaine $t_{20}=1.1, P=0.27$; amplitude, saline versus cocaine $\left.t_{20}=3.7,{ }^{*} P=0.0013\right)$. (d) Images indicating the AAV-GluA1ct injection site, infected $\mathrm{LHb}$ neurons, and GluA1ct-mCherry expression and eGFP labeling in LHb $\rightarrow$ RMTg neurons. (e) Sample mEPSCs obtained in AAV-GluAlct-infected and uninfected $\mathrm{LHb} \rightarrow$ RMTg neurons. (f) Scatter plot and grouped data for mEPSC frequency and amplitude ( $n=6-10,3$ mice of 7-9 weeks; frequency interaction factor $F_{1,36}=0.4, P=0.27$, two-way ANOVA; amplitude interaction factor $F_{1,36}=15.5$, ${ }^{*} P=0.0012$, two-way ANOVA). (g) AMPAR EPSCs $(-70 \mathrm{mV})$ and AMPAR + NMDAR EPSCs (+50 mV) obtained from LHb $\rightarrow$ RMTg neurons. (h) AMPA/NMDA ratios from sequentially recorded neighboring neurons (AAV-GluA1ct infected versus uninfected) after saline or cocaine (AMPA/NMDA; $n=9-11,4$ mice of 7-9 weeks; uninfected saline versus cocaine $t_{18}=2.9$, ${ }^{*} P=0.008$; infected saline versus cocaine $t_{18}=0.4$, $P=0.7$ ). Error bars represent s.e.m.
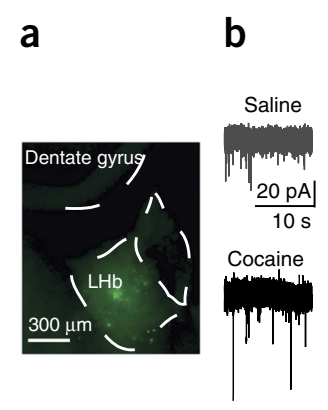

C
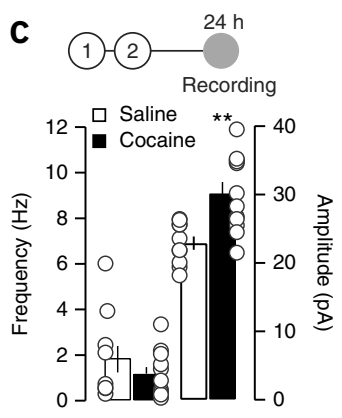

d
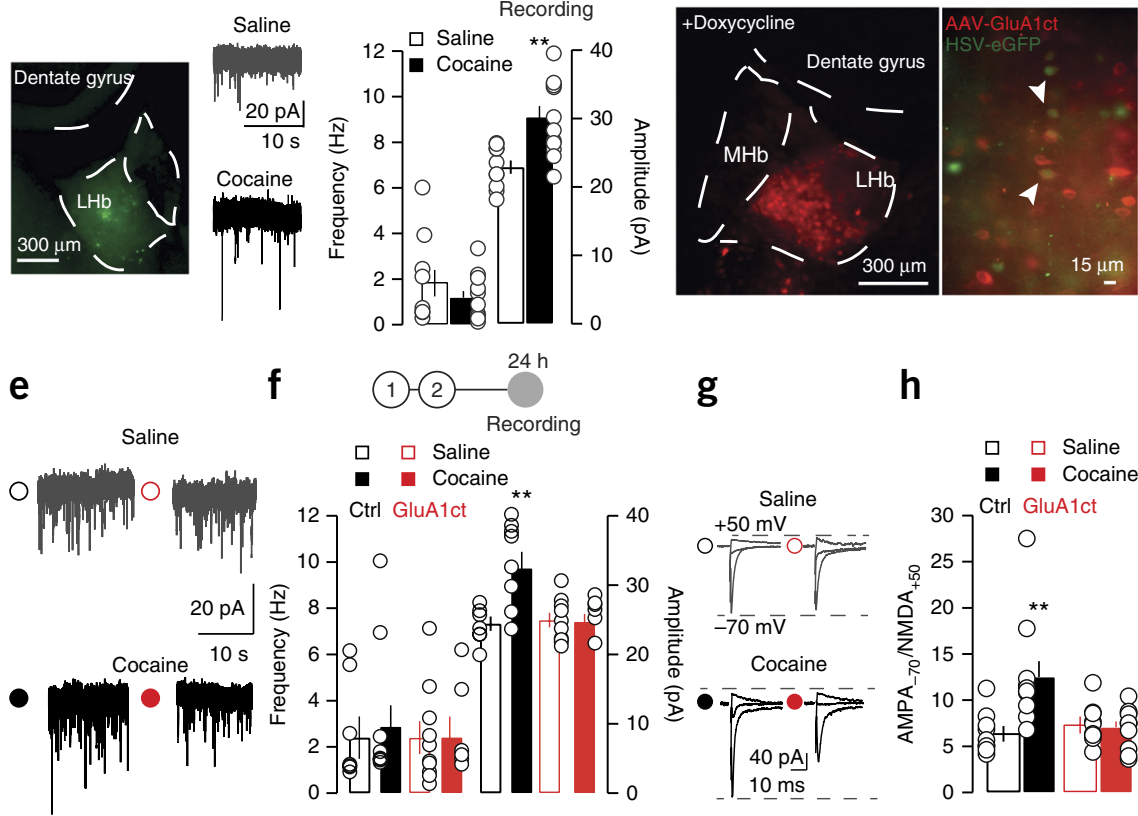

f

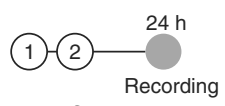

g

h

$\square \quad \square$ Saline ㅁ Saline
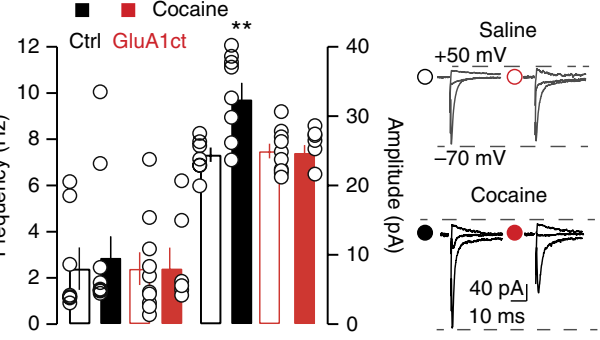

- Cocaine

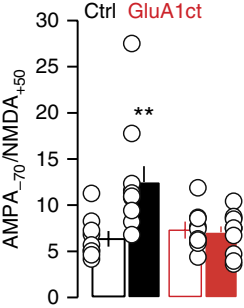

trafficking on LHb neuronal firing (Supplementary Fig. 2i,j). Consistently, we found that input resistance was larger following cocaine treatment, an effect that was prevented by GluAlct expression.

The cell input resistance was highly correlated with $\mathrm{LHb} \rightarrow \mathrm{RMTg}$ neuronal excitability (Fig. 2f,g). To understand the cocaine-driven GluA1dependent mechanisms causing $\mathrm{LHb} \rightarrow \mathrm{RMTg}$ neuron hyperexcitability,

a

Recording: LHb

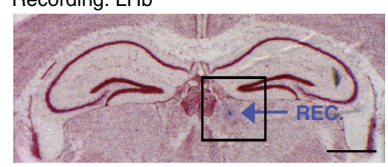

Stimulation: RMTg

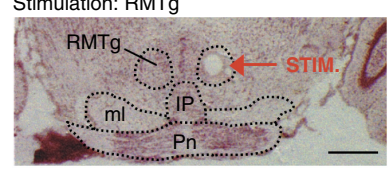

Figure 2 Cocaine drives GluA1-mediated reduction of $\mathrm{K}^{+}$conductance and hyperexcitability in $\mathrm{LHb} \rightarrow \mathrm{RMTg}$ neurons. (a) Recording site in $\mathrm{LHb}$ and stimulation site in RMTg of an anesthetized mouse. (b) Sample of in vivo recordings. (c) Average firing rates from saline- and cocaine-treated mice $(n=7,14,3-4$ mice of 6-8 weeks, $t_{19}=2,{ }^{*} P=0.049$ ). (d,e) Experimental protocol and current-clamp sample traces b c

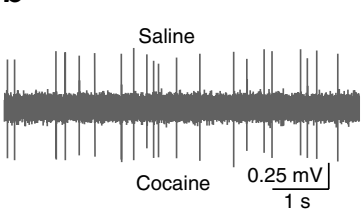

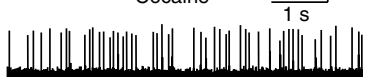

c

d

d Saline $20 \mathrm{mv} /$ cocaine

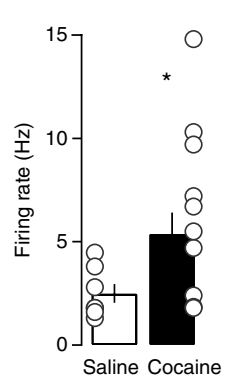

f

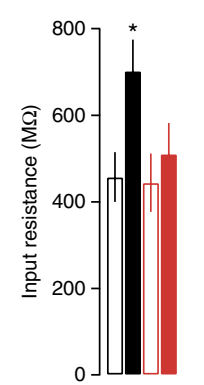

g

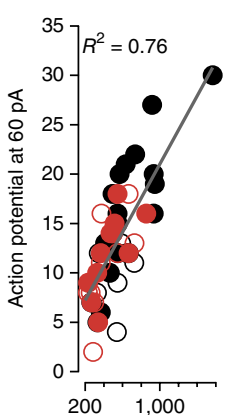

h

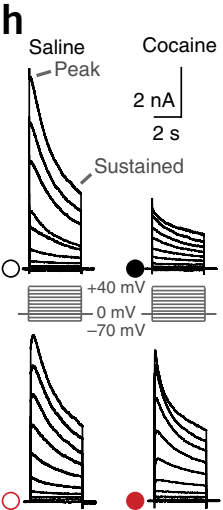

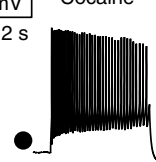

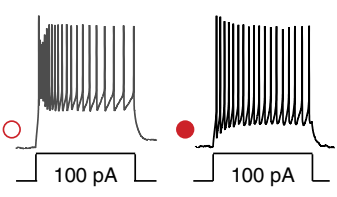

e

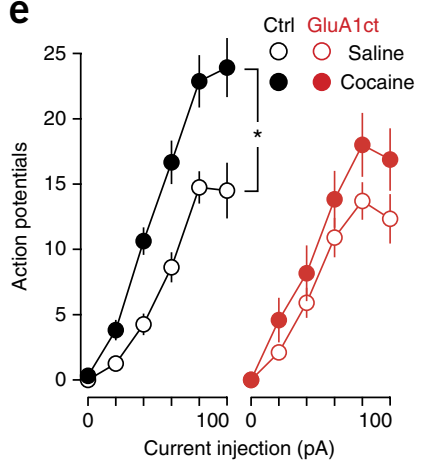

Current injection $(\mathrm{pA})$

(100 pA injection; d) and action potentials evoked by current steps (e) for neighboring AAV-GluAlct-infected and uninfected neurons ( $n=8-16,4$ mice of 7-9 weeks

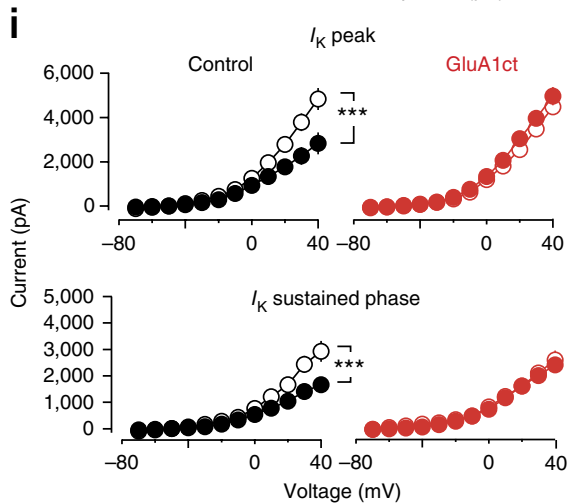

uninfected saline versus cocaine, interaction factor $F_{5,114}=2.8,{ }^{*} P=0.03$ repeated-measures ANOVA; infected saline versus cocaine, interaction factor $F_{5,114}=0.4, P=0.14$ repeated-measures ANOVA). (f) Bar graph for input resistance values obtained in the different experimental groups from neurons recorded in e (input resistance; $n=8-17,6$ mice of 7-9 weeks; uninfected saline versus cocaine $t_{23}=2.1$, ${ }^{*} P=0.04$; infected saline versus cocaine $\left.t_{20}=0.6, P=0.06\right)$. (g) Scatter plot for excitability versus input resistance. (h,i) $\mathrm{K}^{+}$-mediated currents in response to increasing voltage steps of $10 \mathrm{mV}$, for neighboring AAV-GluAlct-infected and uninfected neurons ( $n=12-14,6$ mice of 7-8 weeks; peak current (top) uninfected saline versus cocaine, interaction factor $F_{11,558}=5, * * * P=0.00065$ repeated-measures ANOVA; infected saline versus cocaine, interaction factor $F_{11,287}=0.7$, $P=0.7$ repeated-measures ANOVA; sustained current (bottom) uninfected saline versus cocaine, interaction factor $F_{11,558}=4.8, * * * P=0.0059$ repeated-measures ANOVA; infected saline versus cocaine, interaction factor $F_{11,287}=0.1, P=0.9$ repeated-measures ANOVA). Error bars represent s.e.m. 
a

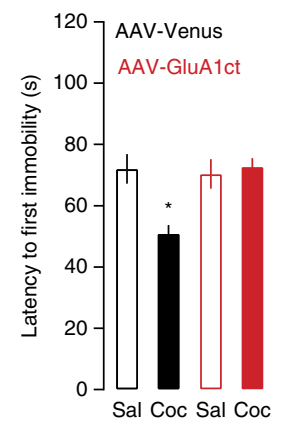

Forced swim test

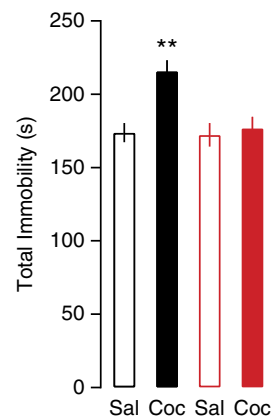

b

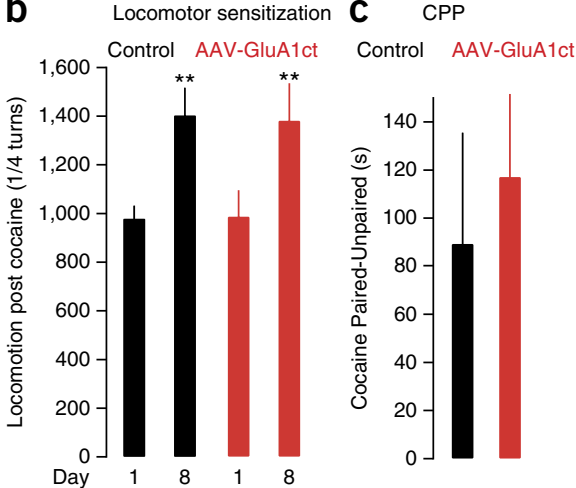

Figure 3 Cocaine-driven AMPAR trafficking in LHb serves for a cocaine-evoked depressive-like state in mice. (a) FST analysis after AAV-GluA1ct and AAV-control injection in LHb ( $n=11-12$ mice of 8-9 weeks; AAV-Venus; latency first immobility $F_{1,44}=3.6,{ }^{*} P=0.038$; total immobility, interaction factor $\left.F_{1,44}=5.9,{ }^{* *} P=0.0011\right)$. (b) Quarter turns in control and AAV-GluA1ct-injected mice ( $n=10$ mice of $8-9$ weeks; paired $t$ test: $t_{9}=3.7{ }^{*} P=0.0044$ for cocaine on day 8 versus day 1 in AAV-control and $t_{9}=3.9 .{ }^{* *} P=0.003$ cocaine on day 8 versus day 1 in AAV-GluAlct-injected mice; $P=0.26$ for locomotor response to cocaine on day 8 for AAV-GluAlct versus control-injected mice). (c) Grouped data for conditioned place preference scores in saline and cocaine-treated animals ( $n=10$ mice of $8-9$ weeks; $\left.t_{18}=0.36 P=0.42\right)$. Error bars represent s.e.m.

we focused on $\mathrm{K}^{+}$conductance, an inhibitory driving force that is critical for neuronal excitability ${ }^{12}$. $\mathrm{LHb}^{\rightarrow \mathrm{RMTg}}$ neurons from cocaine-exposed mice exhibited a marked reduction of $\mathrm{K}^{+}$channelmediated peak and sustained currents, which are mediated by multiple $\mathrm{K}^{+}$channel subtypes (Figs. 1f and $\left.\mathbf{2 h}, \mathbf{i}\right)^{12}$. Similar to the modifications described above, the reduction in $\mathrm{K}^{+}$currents was prevented by the expression of GluA1ct in $\mathrm{LHb} \rightarrow \mathrm{RMTg}$ neurons. This suggests that cocaine triggers a GluA1-dependent synaptic potentiation, resulting in a functional reduction of $\mathrm{K}^{+}$conductance and $\mathrm{LHb} \rightarrow$ RMTg neuron hyperexcitability. Future studies will need to identify the specific $\mathrm{K}^{+}$component underlying cocaine-evoked adaptations of intrinsic excitability.

Is GluA1 trafficking-dependent plasticity in $\mathrm{LHb}$ a requirement for cocaine-driven behavioral phenotypes emerging during withdrawal? We employed the forced swim test (FST) and the tail suspension test (TST) to assess drug-evoked depressive-like states ${ }^{13,14}$. Exposing control mice (AAV-Venus) to five cocaine injections followed by a 2 -week drug-free period resulted in depressive-like behaviors, with reduced latency to first immobility in the FST and increased total immobility in the FST and TST (Fig. 3a and Supplementary Fig. 3a,c). Notably, GluA1ct expression in the LHb prevented the cocaine-induced depressive-like phenotype (Fig. 3a and Supplementary Fig. 3c). Precluding GluA1 trafficking in the LHb specifically abolished the drug-mediated aversive component, as cocaine-induced locomotor sensitization or place preference remained intact following GluA1ct overexpression (Fig. 3b,c and Supplementary Fig. 3b,d,e).

We found that cocaine-evoked GluA1 trafficking mediated the potentiation of AMPAR transmission, reduction of $\mathrm{K}^{+}$conductance and hyperexcitability in the LHb. This ultimately contributes to the emergence of negative depressive-like symptoms during drug withdrawal. The GluA1-driven reduction in $\mathrm{K}^{+}$conductance provides a previously unidentified mechanism, potentially representing a common substrate for disorders characterized by $\mathrm{LHb}$ dysfunction ${ }^{13}$. The cocaine-driven hyperactivity of $\mathrm{LHb} \rightarrow \mathrm{RMTg}$ neurons may provide an 'anti-reward' signal' ${ }^{1}$ that likely inhibits the

\section{ACKNOWLEDGMENTS}

We thank C. Bellone, M.T.C. Brown, C. Lüscher and J.C. Poncer for discussions and comments on the manuscript. This work was supported by funds from the INSERM Atip-Avenir, ERC StG SalienSy 335333 and the Paris School of Neuroscience Network (ENP) to M.M., and the Agence Nationale de la Recherche (ANR-12-bsv4-0022-01) to F. Georges. F.J.M. is supported by a postdoctoral fellowship from the Fyssen Foundation and K.V. is supported by a PhD fellowship from the French Ministry of Science (ESR).

\section{AUTHOR CONTRIBUTIONS}

F.J.M., K.V., S.L. and M.M. performed and analyzed all of the in vitro electrophysiological recordings. K.V., L.M.-P., and M.M. designed and performed the behavioral experiments. I.M. and M.J.M., prepared and tested the viral construct. S.M. and F. Gardoni performed the immunoblots. R.L.H. provided the phospho-mutant mice. F. Georges performed the in vivo recordings. M.M. designed the study and wrote the manuscript with the help of F.J.M., S.L. and K.V.

\section{COMPETING FINANCIAL INTERESTS}

The authors declare no competing financial interests.

Reprints and permissions information is available online at http://www.nature.com/ reprints/index.html.

1. Koob, G.F. \& Le Moal, M. Phil. Trans. R. Soc. Lond. B 363, 3113-3123 (2008).

2. Barr, A.M., Markou, A. \& Phillips, A.G. Trends Pharmacol. Sci. 23, 475-482 (2002).

3. Stamatakis, A.M. \& Stuber, G.D. Nat. Neurosci. 15, 1105-1107 (2012)

4. Jhou, T.C., Fields, H.L., Baxter, M.G., Saper, C.B. \& Holland, P.C. Neuron 61 786-800 (2009).

5. Li, B. et al. Nature 470, 535-539 (2011).

6. Maroteaux, M. \& Mameli, M. J. Neurosci. 32, 12641-12646 (2012).

7. Zuo, W., Chen, L., Wang, L. \& Ye, J.H. Neuropharmacology 70, 180-189 (2013).

8. Meye, F.J., Lecca, S., Valentinova, K. \& Mameli, M. Front. Hum. Neurosci. 7, 860 (2013).

9. Rumpel, S., LeDoux, J., Zador, A. \& Malinow, R. Science 308, 83-88 (2005).

10. Liu, B., Wang, S., Brenner, M., Paton, J.F. \& Kasparov, S. J. Gene Med. 10 583-592 (2008).

11. Malinow, R. Phil. Trans. R. Soc. Lond. B 358, 707-714 (2003)

12. Friedman, A.K. et al. Science 344, 313-319 (2014).

13. Li, K. et al. Science 341, 1016-1020 (2013).

14. Porsolt, R.D., Bertin, A. \& Jalfre, M. Arch. Int. Pharmacodyn. Ther. 229, 327-336 (1977).

15. Matsumoto, M. \& Hikosaka, O. Nature 447, 1111-1115 (2007).

16. Jhou, T.C. et al. J. Neurosci. 33, $7501-7512$ (2013).

17. Weiss, F., Markou, A., Lorang, M.T. \& Koob, G.F. Brain Res. 593, 314-318 (1992). 


\section{ONLINE METHODS}

Surgery. C57Bl/6J wild-type mice, S845A knock-in mice and S831A knock-in mice (males of 4-9 weeks) were used in accordance with the guidelines of the French Agriculture and Forestry Ministry for handling animals. Animals were anesthetized with ketamine ( $90 \mathrm{mg}$ per $\mathrm{kg}$ )/xylazine ( $15 \mathrm{mg}$ per kg intraperitoneal) (Sigma-Aldrich) before bilateral injection with a herpes simplex virus (McGovern Institute) expressing enhanced GFP allowing for retrograde tracing, into the RMTg or VTA. Retrobeads (Lumafluor) were used for recordings performed 2 weeks after withdrawal ${ }^{18}$. The following coordinates were used (from bregma, in mm) ${ }^{18}$ : RMTg: A-P, -2.9 ; M-L, \pm 0.5 ; D-V, -4.3 ; VTA: A-P, $-2.5 ; \mathrm{M}-\mathrm{L}, \pm 0.8 ; \mathrm{D}-\mathrm{V},-4.4$. Recovery was allowed for $5-15 \mathrm{~d}$. When indicated, an adeno-associated virus (AAV) coding for an 89 amino-acid sequence of the GluA1 carboxy-terminal tail was used (AAV-GluA1ct) ${ }^{19}$. The GluA1ct sequence is fused to mCherry and inserted under the control of a TET-ON system. AAVGluA1ct-mCherry and AAV-Venus as a control were injected bilaterally in the $\mathrm{LHb}$ at a final volume of $300-500 \mathrm{nl}$. The following coordinates were used (from bregma, in mm): A-P, -1.7 ; M-L, $\pm 0.45 ; \mathrm{D}-\mathrm{V},-3.1$. After 3 weeks, mice were subjected to doxycycline treatment that consisted of intragastric administrations of $5 \mathrm{mg}$ per $100 \mu \mathrm{l}$ twice per day for three consecutive days. The injection sites were carefully examined for all electrophysiology experiments and only animals with correct injections were used for recordings. Similarly, for behavioral studies only animals with correct injection sites were included in the analysis. Brain slices from mice injected with AAVs or HSV were directly examined under a fluorescent microscope.

Electrophysiology. Animals injected with saline or cocaine $(20 \mathrm{mg}$ per kg, intraperitoneal), once per day for 2 or 5 consecutive days, anesthetized (ketamine/ xylazine; $50 \mathrm{mg} / 10 \mathrm{mg} \mathrm{kg}^{-1}$ intraperitoneal) $24 \mathrm{~h}, 7 \mathrm{~d}$ or $14 \mathrm{~d}$ after the last injection for preparation of $\mathrm{LHb}$-containing brain slices. Slicing was done in bubbled icecold $95 \% \mathrm{O}_{2} / 5 \% \mathrm{CO}_{2}$-equilibrated solution containing (in $\mathrm{mM}$ ): choline chloride 110; glucose 25; $\mathrm{NaHCO}_{3} 25 ; \mathrm{MgCl}_{2} 7$; ascorbic acid 11.6; sodium pyruvate 3.1; $\mathrm{KCl} 2.5 ; \mathrm{NaH}_{2} \mathrm{PO}_{4} 1.25 ; \mathrm{CaCl}_{2}$ 0.5. Coronal slices $(250 \mu \mathrm{m})$ were stored at $\sim 22^{\circ} \mathrm{C}$ in $95 \% \mathrm{O}_{2} / 5 \% \mathrm{CO}_{2}$-equilibrated artificial cerebrospinal fluid (ACSF) containing (in $\mathrm{mM}$ ): $\mathrm{NaCl} 124 ; \mathrm{NaHCO}_{3} 26.2$; glucose $11 ; \mathrm{KCl} 2.5 ; \mathrm{CaCl}_{2} 2.5 ; \mathrm{MgCl}_{2}$ 1.3; $\mathrm{NaH}_{2} \mathrm{PO}_{4} 1$. Recordings (flow rate of $2.5 \mathrm{ml} \mathrm{min}{ }^{-1}$ ) were made under an Olympus-BX51 microscope (Olympus) at $32^{\circ} \mathrm{C}$. Currents were amplified, filtered at $5 \mathrm{kHz}$ and digitized at $20 \mathrm{kHz}$. Access resistance was monitored by a step of $-4 \mathrm{mV}(0.1 \mathrm{~Hz})$. Experiments were discarded if the access resistance increased more than $20 \%$. mEPSCs were recorded in voltage-clamp mode at $-60 \mathrm{mV}$ in presence of picrotoxin $(100 \mu \mathrm{M})$ and tetrodotoxin (TTX, $1 \mu \mathrm{M})$ from retrogradely labeled RMTg-projecting LHb neurons. The internal solution contained (in $\mathrm{mM}$ ): $\mathrm{CsCl} 130 ; \mathrm{NaCl} 4 ; \mathrm{MgCl}_{2}$ 2; EGTA 1.1; HEPES 5; $\mathrm{Na}_{2} \mathrm{ATP} 2$; sodium creatine-phosphate $5 ; \mathrm{Na}_{3} \mathrm{GTP} 0.6$; spermine 0.1 . The liquid junction potential was $-3 \mathrm{mV}$. Neighboring neurons positive for the EGFP retrograde labeling and negative or positive for the mCherry GluA1ct expression were recorded sequentially, counterbalancing the order of patching. EPSCs were evoked through glass electrodes placed $200 \mu \mathrm{m}$ from the recording site. In the case of the sequential recording of neurons, the stimulation electrode was left in place using the same intensity. AMPA:NMDA ratios of evoked-EPSC were obtained by AMPA-EPSC $-70 \mathrm{mV} / \mathrm{NMDA}$-EPSCs at $+50 \mathrm{mV}$ as previously described, using the late component of the EPSC at $30 \mathrm{~ms}$ after the onset ${ }^{18}$. Rectification indexes were computed by AMPA-EPSC $_{-70} /$ AMPA-EPSC $_{+50}$. Current-clamp recordings were obtained using an internal solution containing (in $\mathrm{mM}$ ): potassium gluconate $140 ; \mathrm{KCl} 5$; HEPES 10; EGTA 0.2; $\mathrm{MgCl}_{2} 2 ; \mathrm{Na}_{2} \mathrm{ATP} 4 ; \mathrm{Na}_{3} \mathrm{GTP} 0.3 \mathrm{mM}$; creatine phosphate 10. Current-clamp experiments were performed by a series of current steps (negative to positive) injected to induce action potentials (20-pA injection current per step, $500 \mathrm{~ms}$ ). To isolate voltage-gated $\mathrm{K}^{+}$channel-mediated currents, 4-s incremental $10-\mathrm{mV}$ voltage steps were given from -70 to +40 starting at $-50 \mathrm{mV}$ holding potential in ACSF containing $1 \mu \mathrm{M}$ tetrodotoxin, $200 \mu \mathrm{M} \mathrm{CdCl}_{2}, 20 \mu \mathrm{M}$ NBQX, $100 \mu \mathrm{M}$ AP5 and $100 \mu \mathrm{M}$ picrotoxin.

In vivo recordings in anesthetized mice. Stereotaxic surgery for electrophysiology experiments was performed under isoflurane anesthesia as previously described $^{20}$. Recording pipettes and stimulating electrodes were inserted into the LHb and RMTg, respectively.

A glass micropipette (tip diameter $=2-3 \mu \mathrm{m}, 4-6 \mathrm{M} \Omega$ ) filled with a $2 \%$ pontamine sky blue solution (wt/vol) in $0.5 \mathrm{M}$ sodium acetate was lowered into the
$\mathrm{LHb}$. LHb neurons were identified according to recently established electrophysiological features ${ }^{21}$, these include a broad triphasic extracellular spike $(>3 \mathrm{~ms})$, and a tonic regular, tonic irregular or bursting spontaneous activity. Through these electrodes, the extracellular potential was recorded with an Axoclamp2B amplifier in the bridge mode. The extracellular potential amplified tenfold by the Axoclamp2B amplifier was further amplified 100-fold and filtered (low-pass filter at $300 \mathrm{~Hz}$ and high-pass filter at $0.5 \mathrm{kHz}$ ) via a differential $\mathrm{AC}$ amplifier (model 1700, A-M Systems). Single neuron spikes were discriminated and digital pulses were collected on-line using a laboratory interface and software (CED 1401, SPIKE 2, Cambridge Electronic Design).

Electrical stimulation of the RMTg was used to test for antidromic activation of LHb neurons using high frequency following collision methods, as described previously ${ }^{20}$. A bipolar concentric stimulation electrode was inserted into the RMTg and driven impulses were considered antidromic if they met the following criteria: 1) constant latency of spike response, 2) driving by each paired stimulus pulses at frequencies of $100 \mathrm{~Hz}$ or greater, and 3) collision of driven spikes by spontaneous impulses occurring in a critical interval approximately equal to the sum of the refractory period plus the driving latency.

At the end of each recording experiment, the electrode placement was marked with an iontophoretic deposit of pontamine sky blue dye $(-20 \mu \mathrm{A}$, continuous current for $12-15 \mathrm{~min}$ ). To mark electrical stimulation sites, $10 \mu \mathrm{A}$ of positive current was passed through the stimulation electrode for $1 \mathrm{~min}$ (Fig. 3). After the experimental procedures, the animals were deeply anesthetized with halothane $(5 \%, \mathrm{vol} / \mathrm{vol})$ and decapitated. Brains were removed and snap-frozen in a solution of isopentane at $-70^{\circ} \mathrm{C}$. The tissue was sectioned into $25-\mu \mathrm{m}$-thick coronal slices, mounted, and stained with neutral red to enable histological determination of recording and stimulating electrodes sites.

Viral construct. To construct pAAV-TRE-GluA1ctmCherry-CMV-Tet-On3G, sequential subclonings were performed as described below. PCR primers and synthetic genes were purchased from Eurofins MWG Operon. TRE-GFP was PCR amplified with Phusion High Fidelity DNA Polymerase (ThermoScientific) using pTRE-GFP as template (Clontech). Product was digested with NsiI and SpeI enzyme (New England BioLabs) and cloned into NsiI/SpeI sites of the pCMV-Tet-On3G vector (Clontech) using T4 DNA ligase (Invitrogen), resulting in pTRE-GFP-CMV-Tet-On3G. GluAct DNA (was obtained by gene synthesis of mouse GluA1 nucleotides 2,425 to 2,667 corresponding to amino acids 809 to 889 (ref. 19). GluAlct was cloned in pCMV-mCherry (kindly provided by $\mathrm{X}$. Nicol, Institut de la Vision) in frame with N-terminal mCherry. GluAlctmCherry was then PCR amplified and digested with AgeI and EcoRV enzymes. pTRE-GFP-CMV-Tet-On3G was digested with AgeI and EcoRV enzymes and GluA1ct-mCherry was cloned in place of GFP to get pTRE-GluA1ct-mCherryCMV-Tet-On3G. pAAV-hsyn-hChR2mcherry (Addgene) was cut with MluI and EcoRV to remove hSyn-hChR2mcherry and obtain pAAV empty vector. A linker sequence containing PpumI and AvrII sites was inserted into MluI and EcoRV sites by oligonucleotides annealing and ligation. pTRE-GluA1ct-mCherry-CMVTet-On3G was digested with PpumI and AvrII and the TRE-GluA1ctmCherryCMV-Tet-On3G cassette was ligated into PpumI and AvrII sites of the pAAV vector. Then, the WPRE sequence was removed from $3^{\prime}$ of Tet-On3G by digestion with RsrII and ClaI, filling in and self-ligation of the vector. WPRE was finally cloned in $3^{\prime}$ of the GluA1ct-mCherry into NheI and SpeI sites to obtain pAAV-TRE-GluR1ctmCherry-CMV-Tet-On3G. All constructs were confirmed by restriction and sequence analysis. All of the AAV plasmids were made into recombinant AAV2/5 particles by the Vector Core Facility at the University of North Carolina, Chapel Hill, USA.

Immunoblotting. Treated and control LHb were initially microdissected and stored at $-80{ }^{\circ} \mathrm{C}$ until processing. Tissue was homogenized by ten strokes in a Teflon-glass homogenizer in ice-cold $0.32 \mathrm{M}$ sucrose containing $1 \mathrm{mM}$ HEPES, $1 \mathrm{mM} \mathrm{MgCl}_{2}, 1 \mathrm{mM} \mathrm{NaHCO}_{3}, 0.5 \mathrm{mM} \mathrm{NaF}$ and $0.1 \mathrm{mM}$ phenylmethylsulfonyl fluoride, in the presence of a complete set of protease inhibitors (Complete, Roche Diagnostic, Basel, Switzerland) and phosphatases inhibitors (Sigma Aldrich). Proteins were quantified using Bradford assay (Bio-Rad Laboratories) and $20 \mu \mathrm{g}$ per sample were loaded on 7\% SDS-PAGE gel and transferred to nitrocellulose membrane using Trans-Blot Turbo Transfer System (Bio-Rad Laboratories) to perform western blot analysis. Membranes were blocked with iBlock-TBS (Invitrogen, T2015) for at least $45 \mathrm{~min}$ and subsequently exposed to primary 
antibody overnight at $4{ }^{\circ} \mathrm{C}$ in iBlock-TBS. The next day, membranes were washed in Tris-buffered saline/Tween 20 (TBST) three times for at least $10 \mathrm{~min}$ at $\sim 22^{\circ} \mathrm{C}$, then incubated with horseradish peroxidase (HRP)-coupled secondary antibody (Bio-Rad Laboratories) for $1 \mathrm{~h}$ at $\sim 22^{\circ} \mathrm{C}$ and washed again as before. For detection, Clarity Western ECL Substrates (Bio-Rad Laboratories) were used, and chemiluminescence was exposed to trans-UV (302 nm) with Chemidoc MP System (Bio-Rad Laboratories). We used antibodies to phospho-GluA1 (Ser845) (Millipore, clone EPR2148, 1:1,000), phospho-GluA1 (Ser831) (Millipore, clone N453, 1:1,000) $\alpha$-Tubulin (Sigma-Aldrich, clone DM1A, 1:10,000).

Behavioral assays. All behavioral tests were conducted during the light phase (8:00-19:00). Animals were randomly assigned to the saline or cocaine group after viral injection.

Locomotor sensitization. Procedure was done as described earlier ${ }^{22}$. The locomotor activity was measured in cylindrical locomotor activity boxes (Imetronics, Pessac, France) in a low luminosity environment. Mice previously injected with the AAV-GluAlct-mcherry in the LHb, were treated with doxycycline as described above. $1 \mathrm{~d}$ after the last doxycycline exposure, experimental day 1 , locomotor activity was recorded $30 \mathrm{~min}$ before and $1 \mathrm{~h}$ after cocaine injection (20 mg per kg, intraperitoneal). 1 week after (day 8), each mouse was injected as on day 1 , in the same activity box. Locomotor activity was measured automatically as the number of consecutive beam breaks (one beam per quadrant).

Conditioned place preference. Conditioned place preference was performed in two compartments of the apparatus (Imetronic) with different visual cues and different wall textures, in a low luminosity environment. On day 1 , mice were placed in the center of the apparatus and allowed to explore freely both compartments for $15 \mathrm{~min}$. Then mice of each group were assigned with one or the other compartment in a counterbalanced way to avoid any initial bias in preference. On days 2 , 4 and 6 mice were injected with cocaine $20 \mathrm{mg}$ per $\mathrm{kg}$ and placed immediately in the assigned closed compartment for $20 \mathrm{~min}$. On days 3,5 and 7 the mice were placed in the other closed compartment after saline injection $10 \mathrm{ml} \mathrm{kg}^{-1}$. Finally on day 8 , mice were tested for preference during $15 \mathrm{~min}$ of free exploration and the time they spent in each compartment was automatically recorded. Offline analysis was performed blind to the animal treatments.

Forced swim test. Forced swim test was conducted under normal light condition as previously described ${ }^{23}$. Mice were placed in a cylinder of water $\left(23-25^{\circ} \mathrm{C}\right.$,
$14 \mathrm{~cm}$ in diameter, $27 \mathrm{~cm}$ in height for mice) for $6 \mathrm{~min}$. The depth of water was set to prevent animals from touching the bottom with their hind limbs. Animal behavior was videotracked from the top (Viewpoint). The latency to the first immobility event and the immobility time of each animal spent during the test was counted online by two independent observers blind to the animal treatments. Immobility was defined as floating or remaining motionless, which means absence of all movement except motions required to maintain the head above the water.

Tail suspension test. The tail suspension test was used to assess behavioral despair and conducted under normal light. Mice were suspended by their tails with adhesive tape for a single session of $6 \mathrm{~min}$. Animal behavior was videotracked from the top/front (Viewpoint) and video recorded. Immobility time of each animal spent during the test was counted online by two independent observer blind of the animal treatments. Mice were considered immobile only when they hung passively and motionless.

Analysis and drugs. All drugs were obtained from Abcam and Tocris and dissolved in water, except for TTX (citric acid 1\%, wt/vol) and cocaine $\mathrm{HCl}(\mathrm{NaCl}$ $0.9 \%, \mathrm{vol} / \mathrm{vol})$. Analysis for the electrophysiology data was performed in blocks depending on the experiment. Online/offline analysis were performed using IGOR-6 (Wavemetrics) and Prism (Graphpad). Data analysis for in vivo electrophysiology was performed off-line using Spike2 (CED) software. Sample size required for the experiment was empirically tested by running pilots experiments in the laboratory. Data distribution was assumed to be normal, and single data points are always plotted. Frequency histograms and mean firing rate were determined for each recorded cells. Compiled data are expressed as mean \pm s.e.m. Significance was set at $P<0.05$ using Student's $t$ test, one- or two-way ANOVA with multiple comparison when applicable.

A Supplementary Methods Checklist is available.

18. Maroteaux, M. \& Mameli, M. J. Neurosci. 32, 12641-12646 (2012).

19. Rumpel, S., LeDoux, J., Zador, A. \& Malinow, R. Science 308, 83-88 (2005).

20. Glangetas, C. et al. J. Neurosci. 33, 19657-19663 (2013).

21. Kowski, A.B., Veh, R.W. \& Weiss, T. Neuroscience 161, 1154-1165 (2009).

22. Valjent, E. et al. Neuropsychopharmacology 35, 401-415 (2010).

23. Porsolt, R.D., Bertin, A. \& Jalfre, M. Arch. Int. Pharmacodyn. Ther. 229, 327-336 (1977). 